\title{
Originals
}

\section{The effect of metabolic control on leucine metabolism in Type 1 (insulin-dependent) diabetic patients}

\author{
A. M. Umpleby ${ }^{1}$, M.A. Boroujerdi' ${ }^{2}$, P. M. Brown ${ }^{1}$, E. R. Carson ${ }^{2}$ and P. H. Sönksen ${ }^{1}$ \\ ${ }^{1}$ Department of Medicine, St. Thomas' Hospital and '2Department of Systems Science, The City University, London, UK
}

\begin{abstract}
Summary. Leucine production rate, metabolic clearance rate and oxidation rate were measured in 10Type 1 (insulin-dependent) diabetic patients after (1) $24 \mathrm{~h}$ insulin withdrawal, (2) conventional insulin therapy and (3) an overnight insulin infusion to maintain normoglycaemia, and in 10 control subjects. In the insulin-withdrawn patients, leucine concentration $(259 \pm 17 \mu \mathrm{mol} / 1)$, production rate $\left(2.65 \pm 0.29 \mu \mathrm{mol} \cdot \mathrm{min}^{-1}\right.$. $\left.\mathrm{kg}^{-1}\right)$ and oxidation rate $\left(0.69 \pm 0.10 \mu \mathrm{mol} \cdot \mathrm{min}^{-1} \cdot \mathrm{kg}^{-1}\right)$ were significantly greater $(p<0.001 ; p<0.05 ; p<0.005$ respectively) than corresponding values in control subjects $(127 \pm 6$; $1.81 \pm 0.12 ; 0.19 \pm 0.02)$. Following conventional insulin therapy, leucine concentration $(162 \pm 12 \mu \mathrm{mol} / \mathrm{l})$ and oxidation rate $\left(0.43 \pm 0.05 \mu \mathrm{mol} \cdot \mathrm{min}^{-1} \cdot \mathrm{kg}^{-1}\right)$ were lower than after in -
\end{abstract}

sulin withdrawal but were still significantly greater than in control subjects $(p<0.05 ; p<0.005)$. Although leucine concentration, production rate and metabolic clearance rate were normal after an overnight insulin infusion, leucine oxidation rate was still greater than normal $\left(0.34 \pm 0.06 \mu \mathrm{mol} \cdot \mathrm{min}^{-1}\right.$. $\mathrm{kg}^{-1} ; p<0.05$ ). These results suggest that increased leucine concentration in insulin deficiency is due to elevated leucine production rate caused by increased proteolysis, and that leucine concentration is restored to normal by insulin treatment.

Key words: Leucine turnover, diabetes, insulin protein synthesis, leucine oxidation.
Insulin deficiency in patients with uncontrolled diabetes mellitus results in the breakdown of protein, leading to muscle wasting and increased gluconeogenesis, as described by Aretaeus in $200 \mathrm{AD}$ as the "melting down of the flesh and limbs into sweet urine". Insulin treatment of diabetic patients restores nitrogen balance to normal. It has been shown that plasma concentrations of the branched-chain amino acids are increased in uncontrolled diabetes $[1,2]$, and are decreased to normal levels by insulin treatment $[3,4]$. Since these amino acids are metabolized almost exclusively by extra-hepatic tissues, mainly muscle [5], which forms the major mass of body protein, plasma levels of branched-chain amino acids may reflect the state of protein catabolism in diabetic patients. Studies with muscle isolated from diabetic rats have shown the net release of branched-chain amino acids [6] and the rates of leucine oxidation [7, 8] to be greatly increased. In man, however, Wahren et al. [9] could find no net release of leucine from either the liver or leg muscle of insulin-withdrawn diabetic patients as measured by arterio-venous catheterization despite an elevated plasma leucine concentration. Sherwin et al. [10] found that the clearance of an infusion of leucine was impaired in diabetic patients and restored to normal by insulin treatment. These studies in man therefore suggest that elevated branched-chain amino acid concentrations in diabetes are due to decreased utilization rather than increased production as a result of accelerated protein breakdown.

Since the results of these studies seem incompatible, we have used $1-{ }^{14} \mathrm{C}$ leucine to measure the rate of production, clearance and oxidation of the branched-chain amino acid leucine in insulin-withdrawn diabetic patients, and have examined the effects of insulin treatment. We have also developed a mathematical model of leucine metabolism, and have investigated the effect of insulin treatment on model parameters. Preliminary results have been presented in a Symposium on Metabolic Acidosis [11].

\section{Subjects and methods}

\section{Subjects}

Ten control subjects (aged 18-52) and 10 Type 1 (insulin-dependent) diabetic patients (aged 38-69) were studied. Details are shown in Table 1. All patients were considered to have Type 1 (insulin-dependent) diabetes, but three patients $(1,2$ and 4$)$ had been on tablet treat- 
Table 1. Clinical details of diabetic patients and control subjects

\begin{tabular}{|c|c|c|c|c|c|c|c|c|c|c|}
\hline \multirow{2}{*}{$\begin{array}{l}\text { Diabetic } \\
\text { patients }\end{array}$} & \multirow[t]{2}{*}{ Sex } & \multirow{2}{*}{$\begin{array}{l}\text { Age } \\
\text { (yrs) }\end{array}$} & \multirow{2}{*}{$\begin{array}{l}\text { Height } \\
(\mathrm{cm})\end{array}$} & \multirow{2}{*}{$\begin{array}{l}\text { Weight } \\
(\mathrm{kg})\end{array}$} & \multirow{2}{*}{$\begin{array}{l}\text { Body mass } \\
\text { index }\end{array}$} & \multirow{2}{*}{$\begin{array}{l}\text { Insulin } \\
\text { treatment } \\
(\mathrm{U} / \text { day })\end{array}$} & & \multirow{2}{*}{$\begin{array}{l}\text { Duration of diabetes (years) } \\
\text { complications and other } \\
\text { illness }\end{array}$} & \multicolumn{2}{|l|}{ Diet } \\
\hline & & & & & & & & & Calories & $\begin{array}{l}\text { Protein } \\
\text { (g) }\end{array}$ \\
\hline 1 & M & 65 & 178 & 73.5 & 23 & Actrapid & 2 & 5 & 1545 & 70 \\
\hline 2 & $\mathrm{~F}$ & 52 & 158 & 63.0 & 25 & $\begin{array}{l}\text { Soluble } \\
\text { Isophane }\end{array}$ & $\begin{array}{r}2 \\
12\end{array}$ & 12 & 1085 & 55 \\
\hline 3 & M & 48 & 170 & 67.5 & 23 & $\begin{array}{l}\text { Actrapid } \\
\text { Insulatard }\end{array}$ & $\begin{array}{l}16 \\
30\end{array}$ & $\begin{array}{l}20 \text { Coeliac disease, } \\
\text { Dermatitis, herpetiformis, } \\
\text { Peripheral neuropathy }\end{array}$ & 2305 & 95 \\
\hline 4 & M & 69 & 156 & 62.0 & 26 & Actrapid & 36 & $15^{+}$ & 1530 & 70 \\
\hline 5 & $\mathrm{~F}$ & 46 & 159 & 53.0 & 21 & $\begin{array}{l}\text { Actrapid } \\
\text { Insulatard }\end{array}$ & $\begin{array}{r}8 \\
26\end{array}$ & 9 & 1490 & 70 \\
\hline 6 & M & 42 & 156 & 58.0 & 24 & $\begin{array}{l}\text { Actrapid } \\
\text { Insulatard }\end{array}$ & $\begin{array}{r}2 \\
18\end{array}$ & 12 & 2000 & 95 \\
\hline 7 & M & 38 & 169 & 68.0 & 28 & Insulatard & 48 & 1.5 & 2180 & 100 \\
\hline 8 & M & 55 & 173 & 61.5 & 21 & Mixtard & 24 & $\begin{array}{l}40 \text { Hypothyroid, treated } \\
0.15 \mathrm{mg} \mathrm{day}^{-1} \text { thyroxine }\end{array}$ & 2390 & 105 \\
\hline 9 & M & 38 & 179 & 57.5 & 18 & Insulatard & 58 & 15 & 1905 & 80 \\
\hline 10 & M & 42 & 178 & 69.5 & 22 & Insulatard & 64 & 12 & 1490 & 82 \\
\hline \multicolumn{2}{|c|}{ Mean \pm SEM } & $50 \pm 1$ & $168 \pm 3$ & $63 \pm 2$ & $23 \pm 3$ & & & & & \\
\hline \multicolumn{11}{|c|}{ Control subjects } \\
\hline 1 & $\mathrm{M}$ & 34 & 188 & 84.0 & 24 & & & & & \\
\hline 2 & M & 36 & 178 & 61.0 & 19 & & & & & \\
\hline 3 & M & 35 & 178 & 82.5 & 26 & & & & & \\
\hline 4 & $\mathrm{~F}$ & 52 & 165 & 69.5 & 26 & & & & & \\
\hline 5 & M & 43 & 179 & 92.5 & 29 & & & & & \\
\hline 6 & M & 33 & 180 & 71.0 & 22 & & & & & \\
\hline 7 & $\mathrm{M}$ & 20 & 178 & 76.0 & 24 & & & & & \\
\hline 8 & M & 18 & 175 & 66.5 & 22 & & & & & \\
\hline 9 & M & 44 & 183 & 73.5 & 22 & & & & & \\
\hline 10 & $\mathrm{~F}$ & 45 & 165 & 54.5 & 20 & & & & & \\
\hline \multicolumn{2}{|c|}{ Mean \pm SEM } & $36 \pm 3$ & $177 \pm 2$ & $73 \pm 3$ & $23 \pm 3$ & & & & & \\
\hline
\end{tabular}

ment for 4, 2 and 14 years respectively before being transferred to insulin. They were studied on two separate occasions: (1) following insulin withdrawal $(24 \mathrm{~h}$ after their last dose of insulin [Actrapid, Novo Laboratories, Ltd] which was equivalent to their normal morning insulin dose if they were treated with short-acting insulin, or approximately half their normal insulin if they were treated with intermediate-acting insulin), and (2) after following their conventional insulin treatment for a week and $12 \mathrm{~h}$ after their usual evening insulin dose. Eight of these diabetic patients were also studied after an overnight insulin infusion, with the infusion rate adjusted to maintain their blood glucose between 4 and $6 \mathrm{mmol} / \mathrm{l}$. All patients were on their usual dietary regimes throughout the study. The control subjects had an average daily intake of 2,260 calories with $72.6 \mathrm{~g}$ protein.

All subjects gave written consent. The study was approved by the Ethical Committee of St. Thomas' Hospital, the Isotope Advisory Panel of the Medical Research Council and the Department of Health and Social Security.

\section{Experimental protocol}

All subjects were studied in the morning after a 14-h overnight fast. After cannulation of an antecubital vein for blood sampling, a 30-min rest period was allowed before rapid injection of $25 \mu \mathrm{Ci} 1-{ }^{14} \mathrm{C}$ leucine (L-leucine, $54 \mathrm{mCi} / \mathrm{mmol}$ ) (Radiochemical Centre, Amersham, England) into a contralateral antecubital vein. A bolus injection was administered rather than a constant infusion, as this required a smaller amount of isotope and provided information for the modelling of leucine metabolism. Blood samples were taken immediately before the injection for baseline measurements, then every $\min$ to $5 \mathrm{~min}$, at 8 and $10 \mathrm{~min}$, then every $10 \mathrm{~min}$ to $120 \mathrm{~min}$. Collections of expired air were made at intervals over the $2 \mathrm{~h}$ period via a 2 -way valve into Douglas Bags every $4 \mathrm{~min}$ to $20 \mathrm{~min}$ and every $20 \mathrm{~min}$ thereafter. Inspiratory volume was measured by a Wright Respirometer (British Oxygen Company, Harlow, Essex, England) attached to the inspiratory end of the 2-way value. To accustom patients to the apparatus, they practised using the apparatus before each study. There was no systematic change in $\mathrm{CO}_{2}$ production rate calculated from inspiratory volume and $\% \mathrm{CO}_{2}$ expired in each patient in the three studies, which suggested that the learning period was adequate. The coefficient of variation for measurement of $\mathrm{CO}_{2}$ production was $10.7 \%$.

\section{Analytical methods}

${ }^{14} \mathrm{C}$ leucine was measured on $1-\mathrm{ml}$ plasma samples deproteinized with sulphosalicylic acid, freeze-dried, reconstituted in $250 \mu \mathrm{l}$ water, and separated from other amino acids on a Chromaspek J180 Amino Acid Analyser (Rank Hilger, Margate, Kent, England). Six-minute fractions of column eluate were made, and fractions containing ${ }^{14} \mathrm{C}$ leucine were freeze-dried, reconstituted in $200 \mu \mathrm{l}$ water and counted in BBOT scintillant in a liquid scintillation counter (Sigma Chemical Co, Intertechnique SL 4000). The fraction collected also contained isoleucine, which is an essential amino acid and would therefore not contain ${ }^{14} \mathrm{C}$, and the internal standard nor-leucine. The coefficient of variation of this method on repeated measurements of leucine activity in the same sample was $8.0 \%$. Amino acid concentrations were determined on plasma samples taken every $40 \mathrm{~min}$ throughout the study.

Plasma glucose was measured on a Clandon Scientific glucose analyser (Yellow Springs Instrument Company, OH, USA). Blood 


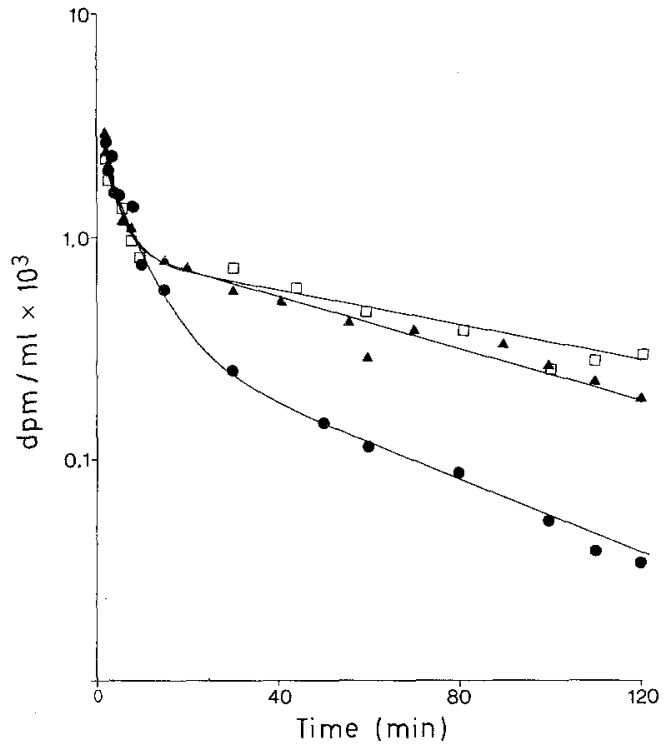

Fig. 1. Plasma ${ }^{14} \mathrm{C}$ leucine time curves fitted with 3 exponentials in diabetic patient 7 following insulin withdrawal $(\square)$, conventional therapy ( $\mathbf{\Delta}$ ) and an insulin infusion ( $\mathbf{O}$

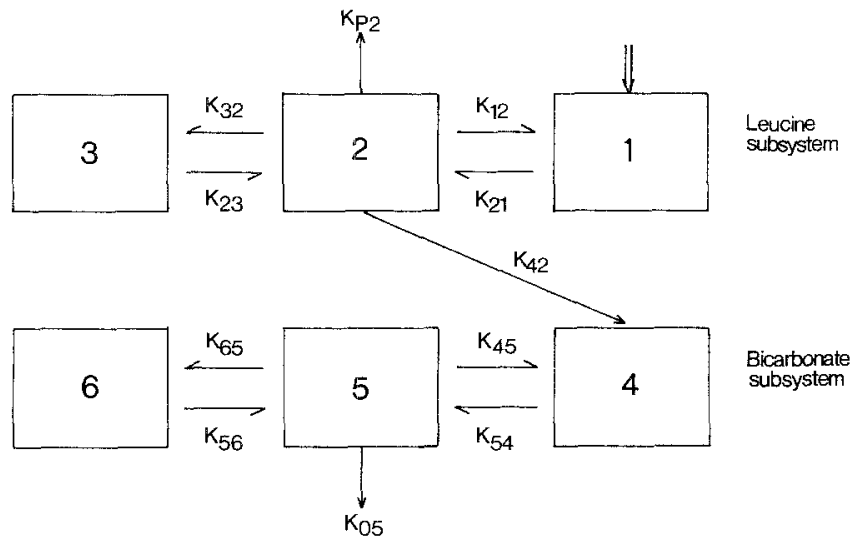

Fig. 2. Compartmental model of leucine and bicarbonate metabolism. The single arrows represent the direction of flux between compartments or out of the system. The double arrow indicates the site of injection

ketones, pyruvate and lactate were determined enzymatically [12, 13]. Plasma non-esterified fatty acids were extracted by the method of Dole and Meinertz [14], and the concentration was determined by the semi-automated method of Carruthers and Young [15]. $\mathrm{HbA}_{1 \mathrm{c}}$ was measured by the method of Baron et al. [16].

Expired ${ }^{14} \mathrm{CO}_{2}$ was measured within $10 \mathrm{~min}$ of sampling by absorbing $0.5 \mathrm{mmol} \mathrm{CO}_{2}$ (after drying over silica gel) into $0.5 \mathrm{ml} 1 \mathrm{M}$ hyamine hydroxide ( $44 \% \mathrm{w} / \mathrm{v}$ ) in $2 \mathrm{ml}$ ethanol using phenolphthalein as an indicator. Ten $\mathrm{ml}$ BBOT scintillant was added, samples were counted in a liquid scintillation counter and $\% \mathrm{CO}_{2}$ was measured on air collected from the Douglas bags using an infra-red capnograph (Godart NV, Bilthoven, Holland).

\section{Analysis of data}

Plasma leucine activity curves $(\mathrm{dpm} / \mathrm{ml})$, as shown in Figure 1, were fitted with the sum of 3 exponentials using a least squares error optimization algorithm [17]. The number of exponentials which produced the "best fit" to the curves (i.e. 3) was determined from a statistical test based on the F-ratio and the distribution of the residual errors [18]. The initial volume of distribution calculated by extrapolation of the curve to $t=0$ was found to approximate a plasma volume of $58.4 \mathrm{ml} /$ $\mathrm{kg}$ body weight [19]. The fitting of the first exponential was dependent on early data points; because on several occasions these were limited by poor blood flow, the fit was constrained by assuming an initial volume of distribution of plasma. This improved the curve fit (i.e. reduced the sum of squares error and improved the distribution of residual errors) in those patients with the paucity of experimental data points in the initial part of the study. A 3-compartment model was postulated to describe leucine kinetics with the number of compartments being determined from the number of exponentials fitting the curve. A model structure was selected which was both mathematically identifiable and physiologically sound. A mamillary structure, a central compartment in equilibrium with 2 peripheral tissue compartments, was not selected since leucine oxidation and protein synthesis would be expected to occur physiologically in both peripheral compartments; this would make the model unidentifiable. The model structure selected was a catenary structure; that is, a plasma compartment in series with two peripheral compartments which represent all tissues which metabolize leucine. This is shown as the leucine subsystem in Figure 2.

Compartment 1 was fixed as plasma volume (as described above). Compartment 2 was selected as the site for leucine oxidation (represented by the rate constant $k_{42}$ in Figure 2$)$ and protein synthesis $\left(k_{p 2}\right)$ as the dynamic response of the compartment was the only one compatible with the ${ }^{14} \mathrm{CO}_{2}$ response observed. Compartment 2 would therefore contain both extracellular and intracellular components. Compartment 3 could not be a site for leucine metabolism, since this would make the model unidentifiable [18]. This compartment may represent an intracellular amino acid pool which is the site of proteolysis but not protein synthesis. This intracellular amino acid pool has been described in several models of amino acid metabolism in isolated tissues $[20,21]$. Net inflow of unlabelled leucine is not shown in Figure 2, since this is a model of distribution and metabolism of labelled material. Since the site of this net inflow was unknown, rates of leucine production, oxidation and rate of leucine incorporation into protein were calculated with the net inflow of unlabelled leucine into compartments 1, 2 and 3. A 3-compartment bicarbonate subsystem based on the model of Steele [22] and adapted by Waterhouse et al. [23], in which the central compartment was the blood pool, was linked to the leucine subsystem. The validation of this linkage is described in the Appendix. The kinetics of labelled leucine and bicarbonate were defined in terms of the following ordinary differential equations:

$$
\begin{aligned}
& \dot{\mathrm{x}}_{1}=\mathrm{k}_{12} \mathrm{x}_{2}-\mathrm{k}_{21} \mathrm{x}_{1} \\
& \dot{\mathrm{x}}_{2}=\mathrm{k}_{21} \mathrm{x}_{1}-\mathrm{k}_{12} \mathrm{x}_{2}-\mathrm{k}_{\mathrm{p} 2} \mathrm{x}_{2}-\mathrm{k}_{42} \mathrm{x}_{2}-\mathrm{k}_{32} \mathrm{x}_{2}+\mathrm{k}_{23} \mathrm{x}_{3} \\
& \dot{\mathrm{x}}_{3}=\mathrm{k}_{32} \mathrm{x}_{2}-\mathrm{k}_{23} \mathrm{x}_{3} \\
& \dot{\mathrm{x}}_{4}=\mathrm{k}_{42} \mathrm{x}_{2}+\mathrm{k}_{45} \mathrm{x}_{5}-\mathrm{k}_{54} \mathrm{x}_{4} \\
& \dot{\mathrm{x}}_{5}=\mathrm{k}_{54} \mathrm{x}_{4}-\mathrm{k}_{05} \mathrm{x}_{5}-\mathrm{k}_{45} \mathrm{x}_{5}-\mathrm{k}_{65} \mathrm{x}_{5}+\mathrm{k}_{56} \mathrm{x}_{6} \\
& \dot{\mathrm{x}}_{6}=\mathrm{k}_{65} \mathrm{x}_{5}-\mathrm{k}_{56} \mathrm{x}_{6}
\end{aligned}
$$

Where $x_{i}, i=1, \ldots 3$, is the activity of ${ }^{14} \mathrm{C}$ labelled leucine in compartments $1, \ldots 3(\mathrm{dpm}) . \mathrm{x}_{j}, \mathrm{i}=4, \ldots 6$ is the activity of ${ }^{14} \mathrm{C}$ labelled bicarbonate in compartments $4, \ldots 6(\mathrm{dpm}) . \mathrm{k}_{\mathrm{ij},}$ =fractional rate constant characterizing transfer of material from compartment $j$ to $i\left(\mathrm{~min}^{-1}\right)$ with initial condition $\mathrm{x}_{1}(0)=1.0$

The observed tracer variables in the experiments are:

$$
\begin{aligned}
& \mathrm{y}_{1}=\mathrm{x}_{1} / \mathrm{V}_{1} \\
& \text { and } \mathrm{y}_{5}=\frac{\mathrm{x}_{5}}{\mathrm{~V}_{5}\left[\mathrm{CO}_{2}\right]} \\
& \text { Where } \mathrm{y}_{1}=\text { plasma }{ }^{14} \mathrm{C} \text { leucine concentration }(\mathrm{dpm} / \mathrm{ml}) \\
& \mathrm{y}_{5}=\text { expired breath } \mathrm{CO}_{2} \text { specific activity }(\mathrm{dpm} / \mathrm{mmol}) \\
& \mathrm{V}_{1}, \mathrm{~V}_{5}=\text { distribution volumes of compartments } 1 \text { and } 5(\mathrm{ml}) \\
& {\left[\mathrm{CO}_{2}\right]=\text { blood } \mathrm{CO}_{2} \text { concentration }(\mathrm{mmol} / \mathrm{ml})}
\end{aligned}
$$

Since both injection of ${ }^{14} \mathrm{C}$ leucine and measurement of leucine activity were in the same compartment, it was not theoretically possible to make unique estimates of all fractional rate constants $\left(\mathrm{k}_{\mathrm{ij}}\right)$ in Eqs. 
(1) to (3). If, however, they were re-parameterized such that $k_{p 2}+k_{42}$ was replaced by $k_{02}$, the model was uniquely identifiable [24]. In this form, estimates were made of the model parameters $\mathrm{k}_{12}, \mathrm{k}_{21}, \mathrm{k}_{23}, \mathrm{k}_{32}$ and $\mathrm{k}_{02}$ (Table 2). Using these model parameters, leucine production rate was estimated with a net input of unlabelled leucine into compartments 1, 2 and 3 (see Appendix).

Leucine production rate

$\mu \mathrm{mol} \cdot \min ^{1} \cdot \mathrm{kg}^{1}$

(calculated as input to compartment 1$)=\frac{V_{1} k_{21} k_{02}[\text { leucine }]}{\left(k_{12}+k_{02}\right) B W}$

Leucine production rate

$\mu \mathrm{mol} \cdot \min ^{1} \cdot \mathrm{kg}^{1}$

(calculated as input to compartments $=\frac{\mathrm{V}_{1} \mathrm{k}_{21} \mathrm{k}_{02} \text { [leucine] }}{\mathrm{K}_{12} \mathrm{BW}}$

2 and 3)

$\mathrm{K}_{12} \mathrm{BW}$

where [leucine] $=$ plasma leucine concentration $(\mu \mathrm{mol} / \mathrm{ml})$

$\mathrm{BW}=$ body weight $(\mathrm{kg})$

The estimate of leucine production rate calculated using Eq. (9) is identical to that obtained by dividing the injected dose of leucine tracer by the area under the leucine specific activity curve [25]; this is the conventional method for calculating leucine production rate.

Table 2. Leucine subsystem parameters in diabetic patients and control subjects (mean $\pm \mathrm{SEM}$ )

\begin{tabular}{lrrrr}
\hline $\begin{array}{l}\text { Parameter } \\
\left(\mathrm{min}^{-1}\right)\end{array}$ & \multicolumn{2}{l}{ Diabetic patients } & \multicolumn{1}{l}{$\begin{array}{l}\text { Control } \\
\text { subjects }\end{array}$} \\
\cline { 2 - 4 } & $\begin{array}{l}\text { Insulin } \\
\text { withdrawal }\end{array}$ & $\begin{array}{l}\text { Conventional } \\
\text { therapy }\end{array}$ & $\begin{array}{l}\text { Insulin } \\
\text { infusion }\end{array}$ & \\
\hline $\mathrm{k}_{12}$ & 0.3980 & 0.3683 & 0.2941 & 0.6103 \\
& \pm 0.0502 & \pm 0.0487 & \pm 0.0689 & +0.1397 \\
$\mathrm{k}_{21}$ & 1.4358 & 1.3788 & 1.1478 & 1.5577 \\
& \pm 0.1785 & \pm 0.1011 & \pm 0.1658 & \pm 0.1494 \\
$\mathrm{k}_{32}$ & 0.1535 & 0.2019 & 0.1959 & 0.2090 \\
& \pm 0.0142 & \pm 0.0445 & \pm 0.0643 & \pm 0.0385 \\
$\mathrm{k}_{23}$ & 0.0789 & 0.0800 & 0.0703 & 0.1570 \\
& \pm 0.0054 & \pm 0.0109 & \pm 0.0204 & \pm 0.0799 \\
$\mathrm{k}_{02}$ & 0.0579 & 0.0923 & 0.1815 & 0.1691 \\
$\left(\mathrm{k}_{42}+\mathrm{k}_{\mathrm{p} 2}\right)$ & \pm 0.0048 & \pm 0.0156 & \pm 0.0892 & \pm 0.0672 \\
\hline
\end{tabular}

Leucine production rate calculated either as input into compartment 2 or as input into compartment 3 (which in the steady state would be identical) may represent a more physiological estimate, since proteolysis would be expected to occur in either compartment 2 or 3 .

Plasma leucine concentration, measured 4 times at 40 -min intervals during each turnover study, remained constant. Thus, steady state was assumed, and the mean of these measurements was used as the value of [leucine] in Eq. (9). The assumption of steady state was made since there was no significant difference by paired Student's t-test between the initial and final measurements of leucine concentration in each turnover study, which were $(\mu \mathrm{mol} / 1$; mean $\pm S E M)$ : in insulinwithdrawn diabetic patients $254 \pm 16$ and $271 \pm 18$; in conventionally treated diabetic patients $158 \pm 10$ and $167 \pm 13$; in insulin-infused diabetic patients $154 \pm 14$ and $140 \pm 10$; and $120 \pm 5$ and $110 \pm 5$ in the control subjects.

The parameter $\mathrm{k}_{05}$ was the rate constant for expired $\mathrm{CO}_{2}$ production. Using this parameter, $\mathrm{CO}_{2}$ production rate can be calculated (see Appendix).

$\mathrm{CO}_{2}$ production rate $=\mathrm{V}_{5}\left[\mathrm{CO}_{2}\right] \mathrm{k}_{05} \mathrm{mmol} / \mathrm{min}$

Leucine oxidation rate was calculated with the input of unlabelled leucine into compartments 1, 2 or 3 from the equation:

Leucine oxidation rate $\left(\mu \mathrm{mol} \cdot \mathrm{min}^{-1} \cdot \mathrm{kg}^{-1}\right)=\frac{\mathrm{k}_{42}}{\mathrm{k}_{42}+\mathrm{k}_{\mathrm{p} 2}} \times$ leucine turnover ( $\mu \mathrm{mol} \cdot \mathrm{min}^{-1} \cdot \mathrm{kg}^{-1}$ ) (calculated as input into compartments 1, 2 or 3) (see Appendix).

Leucine oxidation rate was calculated with the assumption that any recycling of ${ }^{14} \mathrm{CO}_{2}$ into other metabolites was negligible. Ninetyfive per cent recovery of ${ }^{14} \mathrm{CO}_{2}$ has been found following administration of $\mathrm{NaH}^{14} \mathrm{CO}_{3}$ in man (unpublished results).

The rate of leucine incorporation into protein was calculated from leucine turnover minus leucine oxidation rate (with input of unlabelled leucine into compartments 1,2 or 3 ).

\section{Statistical analysis}

All $p$ values were calculated using Student's t-test between control subjects and diabetic patients when the variance of the 2 groups was equal. When variance was unequal, as determined by the F-test, a modified Student's t-test was used [26]. Two-way analysis of variance was used for the calculation of significant differences between the three studies in diabetic patients.

Table 3. Plasma amino acid concentrations in diabetic patients and control subjects

\begin{tabular}{|c|c|c|c|c|c|c|}
\hline \multirow[t]{2}{*}{ Amino acid } & \multicolumn{3}{|l|}{ Diabetic patients } & \multirow{2}{*}{$\begin{array}{l}\text { Control } \\
\text { subjects } \\
\mu \mathrm{mol} / 1\end{array}$} & \multicolumn{2}{|l|}{$p$ values } \\
\hline & $\begin{array}{l}\text { Insulin } \\
\text { withdrawal (IW) } \\
\mu \mathrm{mol} / 1\end{array}$ & $\begin{array}{l}\text { Conventional } \\
\text { therapy (CT) } \\
\mu \mathrm{mol} / 1\end{array}$ & $\begin{array}{l}\text { Insulin } \\
\text { infusion (II) } \\
\mu \text { mol/1 }\end{array}$ & & $\begin{array}{l}\text { IW } \\
\text { versus } \\
\text { CT }\end{array}$ & $\begin{array}{l}\text { CT } \\
\text { versus } \\
\text { II }\end{array}$ \\
\hline Threonine & $84 \pm 10$ & $104 \pm 15$ & $96 \pm 14$ & $149 \pm 11$ & - & - \\
\hline Serine & $97+10$ & $103 \pm 11$ & $107 \pm 7$ & $120 \pm 8$ & - & - \\
\hline Proline & $186 \pm 22$ & $191 \pm 29$ & $165 \pm 27$ & $181 \pm 15$ & - & - \\
\hline Glycine & $186 \pm 14 a$ & $249 \pm 22$ & $269 \pm 37$ & $261 \pm 26$ & $p<0.05$ & NS \\
\hline Alanine & $240 \pm 22$ & $206 \pm 20$ & $199 \pm 20 \mathrm{a}$ & $285 \pm 28$ & - & - \\
\hline Valine & $405 \pm 23 b$ & $263 \pm 17 a$ & $240 \pm 18$ & $200 \pm 12$ & $p<0.001$ & NS \\
\hline Methionine & $22 \pm 1$ & $22 \pm 2$ & $21 \pm 2$ & $19 \pm 1$ & - & - \\
\hline Isoleucine & $146 \pm 10 b$ & $96 \pm 12 a$ & $73 \pm 7$ & $59 \pm 3$ & $p<0.05$ & NS \\
\hline Leucine & $259 \pm 17 b$ & $162 \pm 12 \mathrm{a}$ & $144 \pm 13$ & $127 \pm 6$ & $p<0.001$ & NS \\
\hline Tyrosine & $56 \pm 4$ & $57 \pm 4$ & $53 \pm 5$ & $59 \pm 3$ & - & - \\
\hline Phenylalanine & $61 \pm 4$ & $57 \pm 4$ & $59 \pm 3$ & $47 \pm 2$ & - & - \\
\hline Histidine & $86 \pm 4$ & $83 \pm 5$ & $83 \pm 6$ & $74 \pm 4$ & - & - \\
\hline Lysine & $179 \pm 11$ & $187 \pm 12$ & $200 \pm 14$ & $181 \pm 16$ & - & - \\
\hline Ornithine & $173 \pm 30$ & $142 \pm 15$ & $176 \pm 22$ & $122 \pm 8$ & - & - \\
\hline
\end{tabular}

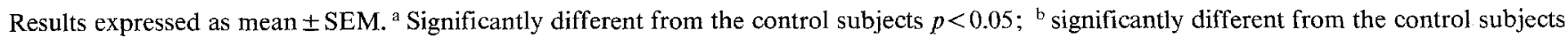
$p<0.001$ 
Table 4. Measurements of leucine MCR, production rate, oxidation rate and the rate of leucine incorporation into protein in diabetic patients and control subjects calculated with the input of unlabelled leucine into compartment 1 (also shown in parentheses are these rates calculated with the input into compartment 2 or 3 )

\begin{tabular}{|c|c|c|c|c|c|c|}
\hline & \multicolumn{3}{|l|}{ Diabetic patients } & \multirow{2}{*}{$\begin{array}{l}\text { Control } \\
\text { subjects }\end{array}$} & \multicolumn{2}{|l|}{$p$ values } \\
\hline & $\begin{array}{l}\text { Insulin } \\
\text { withdrawal (IW) }\end{array}$ & $\begin{array}{l}\text { Conventional } \\
\text { therapy }(\mathrm{CT})\end{array}$ & $\begin{array}{l}\text { Insulin } \\
\text { infusion (II) }\end{array}$ & & $\begin{array}{l}\text { IW versus } \\
\text { CT }\end{array}$ & $\begin{array}{l}\text { CT versus } \\
\text { II }\end{array}$ \\
\hline $\begin{array}{l}\text { Leucine MCR } \\
\qquad\left(\mathrm{ml} \cdot \mathrm{min}^{-1} \cdot \mathrm{kg}^{-1}\right)\end{array}$ & $9.68 \pm 0.37 \mathrm{a}$ & $13.51 \pm 1.07$ & $14.10 \pm 1.35$ & $14.04 \pm 1.36$ & $\mathrm{p}<0.05$ & NS \\
\hline $\begin{array}{l}\text { Leucine } \\
\text { Production } \\
\text { Rate } \\
\qquad\left(\mu \mathrm{mol} \cdot \mathrm{min}^{-1} \cdot \mathrm{kg}^{-1}\right)\end{array}$ & $\begin{array}{c}2.65 \pm 0.29 a \\
(3.07 \pm 0.30 a)\end{array}$ & $\begin{array}{c}2.29 \pm 0.21 \\
(2.80 \pm 0.26)\end{array}$ & $\begin{array}{c}2.00 \pm 0.22 \\
(2.64 \pm 0.28)\end{array}$ & $\begin{array}{c}1.81 \pm 0.12 \\
(2.21 \pm 0.15)\end{array}$ & $\mathrm{p}<0.01$ & $\mathrm{p}<0.05$ \\
\hline $\begin{array}{l}\text { Leucine } \\
\text { Oxidation } \\
\text { Rate } \\
\qquad\left(\mu \mathrm{mol} \cdot \mathrm{min}^{-1} \cdot \mathrm{kg}^{-1}\right)\end{array}$ & $\begin{array}{c}0.69 \pm 0.10 b \\
(0.80 \pm 0.12 b)\end{array}$ & $\begin{array}{c}0.43 \pm 0.05 b \\
(0.53 \pm 0.19 b)\end{array}$ & $\begin{array}{c}0.34 \pm 0.06 a \\
(0.45 \pm 0.07 a)\end{array}$ & $\begin{array}{c}0.19 \pm 0.02 \\
(0.24 \pm 0.03)\end{array}$ & $\mathrm{p}<0.005$ & NS \\
\hline $\begin{array}{l}\text { Rate of Leucine } \\
\text { Incorporation } \\
\text { into Protein } \\
\quad\left(\mu \mathrm{mol} \cdot \mathrm{min}^{-1} \cdot \mathrm{kg}^{-1}\right)\end{array}$ & $\begin{array}{c}1.96 \pm 0.25 \\
(2.10 \pm 0.29)\end{array}$ & $\begin{array}{c}1.85 \pm 0.19 \\
(2.28 \pm 0.23)\end{array}$ & $\begin{array}{c}1.66 \pm 0.18 \\
(2.19 \pm 0.23)\end{array}$ & $\begin{array}{c}1.61 \pm 0.11 \\
(1.98 \pm 0.13)\end{array}$ & $\begin{array}{l}\text { NS } \\
\text { NS }\end{array}$ & $\begin{array}{l}\text { NS } \\
\text { NS }\end{array}$ \\
\hline $\begin{array}{l}\% \text { Difference between } \\
\text { the } 2 \text { methods of } \\
\text { calculation }\end{array}$ & $13.98 \pm 1.73$ & $17.87 \pm 1.91$ & $23.75 \pm 2.86$ & $17.98 \pm 1.94$ & NS & NS \\
\hline
\end{tabular}

Results expressed as mean \pm SEM. ${ }^{\text {a }}$ Significantly different from control subjects $p<0.05 .{ }^{b}$ Significantly different from control subjects $p<0.005$

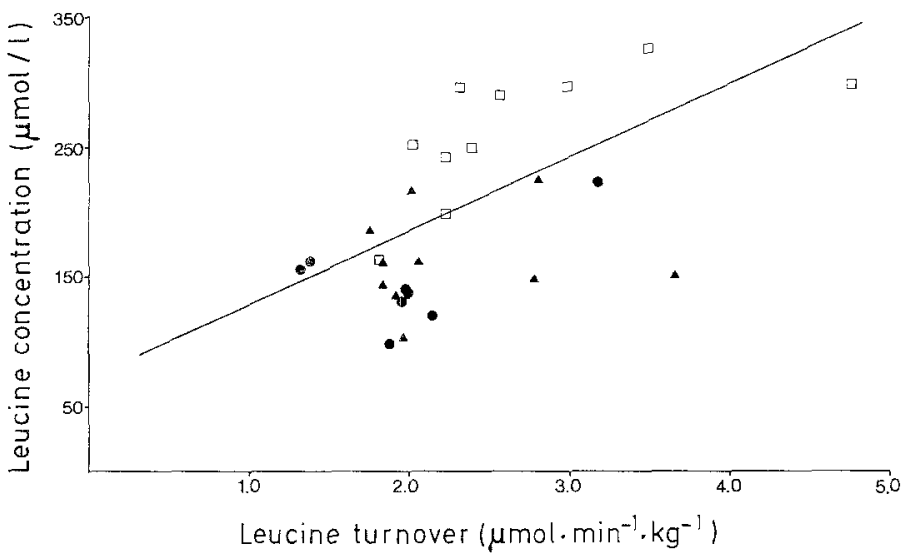

Fig. 3. The relationship between leucine turnover rate and plasma leucine concentration $(r=0.53, p<0.01)$ in diabetic patients following insulin withdrawal $(\square)$, conventional therapy $(\Delta)$ and an insulin infusion (

\section{Results}

Although there were no statistically significant differences between the leucine subsystem parameters in the diabetic patients studied after insulin withdrawal, conventional therapy and insulin infusion, $\mathrm{k}_{02}$ (sum of $\mathrm{k}_{42}+\mathrm{k}_{\mathrm{p} 2}$ ) was found to increase with insulin treatment (Table 2).

Means of the plasma amino acid concentrations measured during each study in control subjects and diabetic patients are shown in Table 3. Leucine, isoleucine and valine concentrations were significantly greater following withdrawal of insulin from diabetic patients than in control subjects $(p<0.001)$. Although the con- centrations of these amino acids were significantly lower when diabetic patients were following their conventional insulin therapy $(p<0.001, p<0.05$ and $p<0.001)$, concentrations were still significantly greater than in control subjects $(p<0.05)$. After an overnight insulin infusion, branched-chain amino acids in diabetic patients were normalized.

Metabolic clearance rate, leucine production rate, oxidation rate and rate of leucine incorporation into protein in control subjects and diabetic patients calculated with input of unlabelled leucine into compartment 1 are shown in Table 4. Following withdrawal of insulin from diabetic patients, leucine metabolic clearance rate was lower than in control subjects $(p<0.05)$. Both conventional insulin therapy and an overnight insulin infusion restored metabolic clearance rate to normal. This increase in metabolic clearance rate with insulin treatment is demonstrated in the model by an increase in the parameter $\mathrm{k}_{02}$ (sum of $\mathrm{k}_{42}+\mathrm{k}_{\mathrm{p} 2}$ ).

Since diabetic patients were in a steady state at the time they were studied, leucine turnover rate was equal to its rate of production and uptake. Leucine production rate was significantly greater in insulin-withdrawn diabetic patients than in control subjects $(p<0.05)$, but was reduced to normal levels by both conventional insulin therapy and an insulin infusion. There was a linear relationship between leucine turnover and leucine concentration in diabetic patients $(r=0.53, p<0.01$; Fig. 3 ).

The rate of leucine oxidation was also greater in insulin-withdrawn diabetic patients than in control subjects $(p<0.005)$. Although this rate was lower after con- 
Table 5. Measurements of blood metabolites in diabetic patients and control subjects

\begin{tabular}{|c|c|c|c|c|c|c|}
\hline & \multicolumn{3}{|l|}{ Diabetic patients } & \multirow{2}{*}{$\begin{array}{l}\text { Control } \\
\text { subjects }\end{array}$} & \multicolumn{2}{|l|}{$p$ values } \\
\hline & $\begin{array}{l}\text { Insulin } \\
\text { withdrawal (IW) }\end{array}$ & $\begin{array}{l}\text { Conventional } \\
\text { therapy }(\mathrm{CT})\end{array}$ & $\begin{array}{l}\text { Insulin } \\
\text { infusion (II) }\end{array}$ & & $\begin{array}{l}\text { IW versus } \\
\text { CT }\end{array}$ & $\begin{array}{l}\text { CT versus } \\
\text { II }\end{array}$ \\
\hline $\begin{array}{l}\text { Acetoacetate } \\
(\mathrm{mmol} / \mathrm{l})\end{array}$ & $\begin{aligned} & 0.433 \mathrm{c} \\
\pm & 0.045\end{aligned}$ & $\begin{aligned} & 0.165 b \\
\pm & 0.032\end{aligned}$ & $\begin{array}{r}0.96 a \\
\pm 0.018\end{array}$ & $\begin{array}{r}0.050 \\
\pm 0.006\end{array}$ & $\mathrm{p}<0.001$ & NS \\
\hline $\begin{array}{l}\text { 3-Hydroxybutyrate } \\
\text { (mmol/l) }\end{array}$ & $\begin{aligned} & 1.520 c \\
\pm & 0.289\end{aligned}$ & $\begin{aligned} & 0.452 \mathrm{a} \\
\pm & 0.143\end{aligned}$ & $\begin{aligned} & 0.221 \mathrm{a} \\
\pm & 0.040\end{aligned}$ & $\begin{array}{r}0.049 \\
\pm 0.007\end{array}$ & $\mathrm{p}<0.05$ & NS \\
\hline $\begin{array}{l}\text { 3-Hydroxybutyrate: } \\
\text { Acetoacetate }\end{array}$ & $\begin{aligned} & 3.514 c \\
\pm & 0.515\end{aligned}$ & $\begin{array}{r}2.223 \\
\pm 0.510\end{array}$ & $\begin{aligned} & 2.900 \mathrm{c} \\
\pm & 0.437\end{aligned}$ & $\begin{array}{r}1.100 \\
\pm 0.200\end{array}$ & NS & NS \\
\hline $\begin{array}{l}\text { Pyruvate } \\
\qquad(\mathrm{mmol} / \mathrm{l})\end{array}$ & $\begin{aligned} & 0.056 \mathrm{c} \\
\pm & 0.006\end{aligned}$ & $\begin{aligned} & 0.043 \mathrm{a} \\
\pm & 0.003\end{aligned}$ & $\begin{array}{r}0.037 \\
\pm 0.004\end{array}$ & $\begin{array}{r}0.035 \\
\pm 0.003\end{array}$ & NS & NS \\
\hline $\begin{array}{l}\text { Lactate } \\
\qquad(\mathrm{mmol} / \mathrm{l})\end{array}$ & $\begin{aligned} & 0.917 \mathrm{~b} \\
\pm & 0.097\end{aligned}$ & $\begin{array}{r}0.582 \\
\pm 0.068\end{array}$ & $\begin{array}{r}0.532 \\
\pm 0.038\end{array}$ & $\begin{array}{r}0.522 \\
\pm 0.124\end{array}$ & $p<0.01$ & NS \\
\hline $\begin{array}{l}\text { Glucose } \\
\qquad(\mathrm{mmol} / 1)\end{array}$ & $\begin{aligned} & 20.26 \mathrm{c} \\
\pm & 0.95\end{aligned}$ & $\begin{array}{l}10.54 \mathrm{a} \\
\pm 1.86\end{array}$ & $\begin{array}{r}5.45 \\
\pm 0.06\end{array}$ & $\begin{array}{r}5.05 \\
\pm 0.11\end{array}$ & $p<0.001$ & $p<0.05$ \\
\hline $\begin{array}{l}\text { Non-esterified } \\
\text { fatty acids (mmol/l) }\end{array}$ & $\begin{aligned} & 1.07 \mathrm{c} \\
\pm & 0.14\end{aligned}$ & $\begin{array}{r}0.67 \\
\pm 0.12\end{array}$ & $\begin{array}{r}0.71 \\
\pm 0.05\end{array}$ & $\begin{array}{r}0.51 \\
\pm 0.05\end{array}$ & NS & NS \\
\hline
\end{tabular}

Results expressed as mean \pm SEM. ${ }^{\text {a }}$ Significantly different from control subjects $p<0.05 ;{ }^{b}$ significantly different from control subjects $p<0.01$; ${ }^{c}$ significantly different from control subjects $p<0.005$

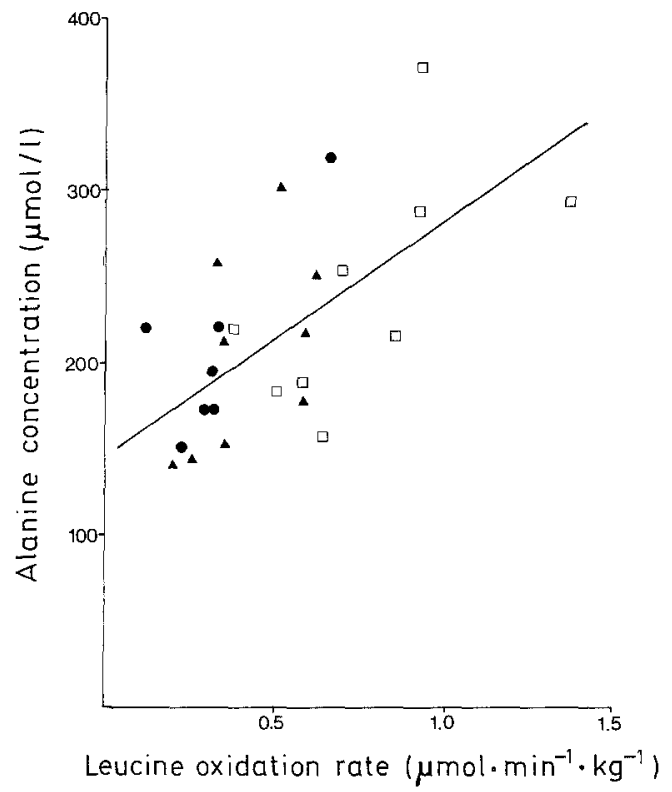

Fig.4. The relationship between leucine oxidation rate and plasma alanine concentration $(r=0.63, p<0.001)$ in diabetic patients following insulin withdrawal $(\square)$, conventional therapy $(\boldsymbol{\Delta})$ and an insulin infusion (๑)

ventional insulin therapy $(p<0.005)$ it was still greater than in control subjects $(p<0.005)$. Unlike all the other measurements of leucine metabolism, oxidation rate was also greater than normal in overnight insulin-infused diabetic patients $(p<0.05)$.

There were no differences between estimates of leucine incorporation into protein in diabetic patients following insulin withdrawal, conventional therapy or an insulin infusion, or between diabetic patients and control subjects.

Also shown in parentheses in Table 4 are the rates of

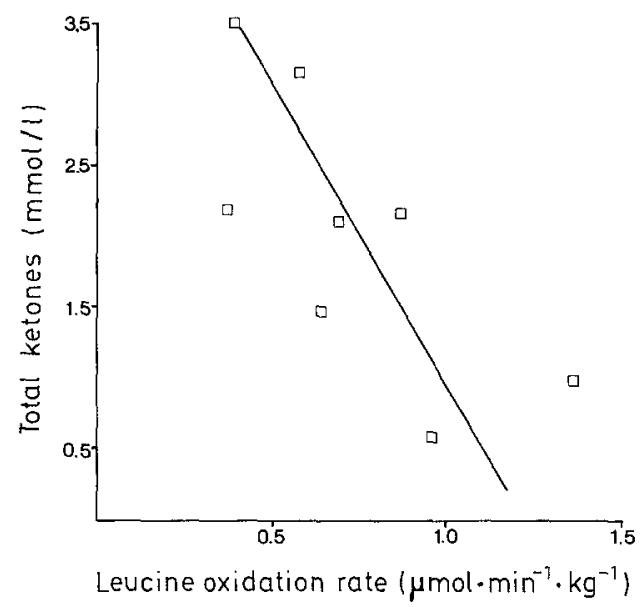

Fig. 5. The inverse relationship between leucine oxidation rate and total ketones in the insulin-withdrawn diabetic patients $(r=-0.72$, $p<0.05$ )

leucine production, oxidation and the rate of leucine incorporation into protein when calculated with the input of unlabelled leucine into compartments 2 or 3 . Although these rates were all higher by $14-24 \%$ than when calculated with the input of unlabelled leucine into compartment 1 (equivalent to the calculation of these rates by non-compartmental analysis) the effect of insulin withdrawal, conventional therapy and insulin infusion on these rates was the same. The degree of diabetic control had no effect on the percentage difference between the rates calculated by these two methods.

Table 5 shows levels of other intermediary metabolites in diabetic patients and control subjects. As expected, acetoacetate and 3-hydroxybutyrate were significantly greater in insulin-withdrawn diabetic patients than in control subjects $(p<0.005)$. Although concentrations were lower in diabetic patients following both 
conventional therapy and an insulin infusion, they were still greater than normal $(p<0.05)$.

In the pooled results from diabetic patients, there was a linear relationship between leucine oxidation rate (leucine input into compartment 1 ) and alanine concentration (Fig. $4, r=0.63, p<0.001$ ).

In the data from insulin-withdrawn diabetic patients, there was an inverse relationship between total blood ketones and leucine oxidation rate (leucine input into compartment $1 ; r=-0.72, p<0.05 ;$ Fig. 5 ). In this group an inverse relationship was also found between total ketones and alanine concentration $(r=-0.71, p<$ 0.05 ). These latter two relationships were not found when diabetic patients were treated with insulin.

$\mathrm{HbA}_{1}$ averaged $11.0 \%$ (range 6.8-14.0\%) in the diabetic patient group, and was not significantly different in the 3 separate studies, i.e. $11.95,10.25$ and 11.0 for insulin withdrawal, conventional therapy and insulin infusion, respectively (normal range: $6-8.5 \%$ ).

\section{Discussion}

In this study we have developed a model to describe leucine kinetics. Leucine production rate, oxidation rate and rate of leucine incorporation into protein were calculated from this model with the input of unlabelled leucine (leucine production rate) into compartment 1. This rate of leucine production is identical to the rate calculated by analysing leucine specific activity curves using non-compartmental analysis. These measurements of rates of leucine production, oxidation and incorporation into protein were similar to values previously determined in control subjects using non-compartmental analysis [27]. Measurements of leucine metabolism were also made with the input of unlabelled leucine (leucine production rate) into compartments 2 and 3 , which at steady state gives identical estimates of production rate. Leucine production and oxidation rate, and incorporation into protein, were all $14-24 \%$ greater than when calculated with the input of unlabelled leucine into compartment 1 . The effect of diabetic control on these estimates, however, was the same with both methods of calculation.

Although whole blood specific activity of leucine could have been measured, we elected to measure plasma leucine specific activity since it is technically more simple and less likely to yield materially different results. [It was assumed that the red blood cell leucine pool was in equilibrium with the plasma pool.] This pool is unlikely to be a significant or even measurable component of leucine dynamics, since red blood cells have lost their nuclei and mitochondria and do not undertake protein synthesis or leucine oxidation.

Measurements of leucine metabolism by non-compartmental analysis were based on the assumption that the plasma specific activity of leucine approximates the intracellular specific activity. Therefore, leucine production rate as measured in plasma should approximate the rate of proteolysis. Proteolysis, however, would be expected to dilute the intracellular specific activity of leucine; this would lead to an underestimation of leucine production rate and hence the rates of leucine oxidation and incorporation into protein which are derived using the production rate. Measurements of intracellular leucine specific activity in a muscle biopsy, following infusion of $1{ }^{13} \mathrm{C}$ leucine, have shown levels to be $88 \%$ of plasma specific activity [28]. Plasma $\alpha$ ketoisocaproate specific activity has been found to be $80 \%$ of plasma leucine specific activity [29] in postabsorptive normal subjects and has been suggested to reflect intracellular leucine specific activity. These values are in keeping with a $14-24 \%$ difference in measurements calculated with 2 different sites of leucine input in the current model. Estimates of leucine kinetics calculated with an input of unlabelled leucine into compartment 2 or 3 may therefore be more physiological estimates. Proteolysis would be expected to occur in either of these two compartments, and the higher rates obtained would be compatible with the lower specific activity reported for intracellular leucine. Since the trends of the measurements of leucine metabolism in the different groups studied were identical, discussion of these two measurements is applicable to either. Although regression analysis used measurements based on an input into compartment 1 , comparable results are obtained with estimates based on an input into compartment 2 and 3.

As previously reported, plasma branched-chain amino acids were elevated in insulin-withdrawn diabetic patients 1,2 and 9 and were restored to normal levels by an overnight insulin infusion. The linear relationship between leucine turnover and concentration indicated that elevated leucine concentration was the result of increased production rate, and was probably due to an increase in proteolysis. Increased proteolysis, however, would be expected to increase the plasma concentrations of all amino acids, yet only the branched-chain amino acids were increased. This may be due to increased hepatic uptake of amino acids, with the exception of branched-chain amino acids, which are not readily utilized by liver [5]. The rate of incorporation of leucine into protein was not significantly different from normal in insulin-withdrawn diabetic patients. These results differ from the study by Nair et al. [27], which showed protein synthesis to be increased in insulinwithdrawn diabetic patients. Both these observations in man appear to conflict with studies which have shown decreased protein synthesis in heart and skeletal muscle of diabetic rats [30, 31 and 32], and a study which has shown a stimulation of synthesis by insulin [30]. In diabetic rat liver, protein synthesis has been shown to be decreased in perfused tissue [33], whereas an in vivo study has shown a decrease in albumin synthesis but no change in the synthesis of other plasma proteins [32]. In 
these studies, protein synthesis was measured in diabetic rats that had either never been treated or were studied several days after insulin withdrawal. The discrepancy between these studies and the present study may be because a longer period of insulin withdrawal is necessary to demonstrate a decrease in protein synthesis. It must also be considered that measurement of protein synthesis in the whole body cannot give any insight into synthesis rates in individual tissues. An increase or normal protein synthesis rate in one tissue such as liver may override any decrease which may occur in another tissue such as muscle, which could result in a normal whole body synthesis rate.

A decrease in leucine metabolic clearance rate also contributed to the elevated leucine concentration in the insulin-withdrawn diabetic patients. While this confirms a previous study which showed the clearance of an unlabelled infusion of leucine to be decreased in insulin-withdrawn diabetic patients [10], it demonstrates that the conclusion that this implied decreased utilization was incorrect. In the present study, the use of isotope turnover techniques showed that, although clearance was decreased, leucine utilization was increased. The decrease in leucine metabolic clearance rate in insulin-withdrawn diabetic patients could be due to insulin lack affecting uptake or subsequent metabolism. Alternatively, the decrease could be the result of an increased leucine concentration causing partial saturation of this clearance process or competition of ketone bodies or other substances with leucine for oxidation and metabolism.

Leucine oxidation was shown to be increased in insulin-withdrawn diabetic patients in agreement with a previous study in diabetic patients [27]. In the present study, although insulin treatment lowered leucine oxidation, it was not reduced to normal levels. This was unlike insulin treatment of previously studied diabetic patients [27] in which leucine oxidation rate was reduced to normal. In rat adipose tissue, where leucine is used as a substrate for fatty acid synthesis, insulin has been found to increase the activity of the branched-chain $\alpha$-keto acid dehydrogenase [34], thus stimulating leucine oxidation. In contrast, leucine oxidation has been shown to be increased in diaphragm muscle [7] and in gastrocnemius muscle, liver and kidney homogenates [8] from diabetic rats, suggesting that insulin may inhibit the branched-chain $\alpha$-keto acid dehydrogenase in these tissues. Since whole body leucine oxidation was increased following insulin withdrawal in diabetic patients, increased oxidation in tissues other than adipose must be considerably greater than any decrease which would be expected to occur in adipose tissue; alternatively, there may be species differences between man and the rat.

There is increasing evidence that oxidation of branched-chain amino acids provides amino groups for synthesis of the major gluconeogenic amino acid alanine $[35,36]$. Alanine is synthesized by the transamination of pyruvate with glutamate, with the major source of glutamate being from transamination of branchedchain amino acids with $\alpha$ ketoglutarate. This hypothesis is demonstrated by the linear relationship between leucine oxidation rate and alanine concentration found in the present study. The inverse relationship between leucine oxidation and total ketones and between alanine concentration and total ketones in insulin-withdrawn diabetic patients provides further evidence for the existence of a ketone-alanine cycle in which an increased supply of ketones decreases alanine production by inhibiting branched-chain amino acid catabolism [37,38].

This study has shown that elevated branched-chain amino acids in uncontrolled diabetes are due to an elevated production rate as a result of increased proteolysis, and that they therefore provide an indication of the state of protein metabolism. Insulin deficiency was also shown to result in an increased leucine oxidation rate, which, in view of its role in alanine synthesis, may contribute to the increased gluconeogenesis found in uncontrolled diabetes $[9,39]$. The observed changes in leucine and protein metabolism in uncontrolled diabetes were probably due directly to the lack of insulin. The large changes in concentrations of glucose, ketones and non-esterified fatty acids and in the altered redox state may, however, also play a role either opposing or accentuating the effect of insulin deficiency. Leucine oxidation for example, may be restrained by the presence of marked ketosis as suggested by this study. Non-esterified fatty acids have been shown to inhibit leucine production rate and leucine oxidation in dogs [40], and in increase in NADH/NAD has been found to increase protein synthesis in isolated rat muscle [41].

We have described a model of leucine kinetics which we have used to calculate leucine turnover and oxidation. The validity of the postulated model structure and its linkage to the bicarbonate subsystem was assessed by comparing a model based estimate of $\mathrm{CO}_{2}$ production rate with independently measured values. We have shown these values to be in close agreement. By developing a model, the rate of leucine production, oxidation and incorporation into protein could be calculated with the input of unlabelled leucine into an intracellular compartment, i.e. 2 or 3 . These rates may be better estimates of the "true" rates than those estimated with an input of leucine into plasma, which is the conventional non-compartmental method of analysis. Although statistically it was not possible to identify insulin-dependent parameters, the development of this model will aid further studies of leucine metabolism. It will be particularly useful in studies which require a complicated analysis of data such as in non-steady state investigations. Since the model can also be used to predict the response of the system to a stimulus, it can help in experimental design. Linkage of the leucine model to models of other substrates and hormones may enable a better understanding of the control of leucine metabolism and its interaction with the metabolism of other substrates. 


\section{Appendix}

The differential equations describing the activity of $1-{ }^{14} \mathrm{C}$ leucine are as follows:

$\dot{\mathrm{x}}_{1}=-\mathrm{k}_{21} \mathrm{x}_{1}+\mathrm{k}_{12} \mathrm{x}_{2}$

$\dot{\mathrm{x}}_{2}=\mathrm{k}_{21} \mathrm{x}_{1}-\mathrm{k}_{12} \mathrm{x}_{2}-\mathrm{k}_{32} \mathrm{x}_{2}-\mathrm{k}_{02} \mathrm{x}_{2}+\mathrm{k}_{23} \mathrm{x}_{3}$

$\dot{\mathrm{x}}_{3}=\mathrm{k}_{32} \mathrm{x}_{2}-\mathrm{k}_{23} \mathrm{x}_{3}$

where $\mathrm{k}_{02}=\mathrm{k}_{42}+\mathrm{k}_{\mathrm{p} 2}$ (see main text)

in matrix form

$$
\left[\begin{array}{l}
\dot{\mathrm{x}}_{1} \\
\dot{\mathrm{x}}_{2} \\
\dot{\mathrm{x}}_{3}
\end{array}\right]=\left[\begin{array}{rcr}
-\mathrm{k}_{21} & \mathrm{k}_{12} & \\
\mathrm{k}_{21} & -\left(\mathrm{k}_{12}+\mathrm{k}_{32}+\mathrm{k}_{02}\right) & \mathrm{k}_{23} \\
& \mathrm{k}_{32} & -\mathrm{k}_{23}
\end{array}\right]\left[\begin{array}{l}
\mathrm{x}_{1} \\
\mathrm{x}_{2} \\
\mathrm{x}_{3}
\end{array}\right]
$$

or

$\dot{\mathrm{x}}=\mathrm{A} \underline{\mathrm{x}}+\mathrm{B} \mathrm{u}$

taking the Laplace transform

$[\mathrm{sI}-\mathrm{A}] \mathrm{x}=\mathrm{Bu}$

$\underline{x}=[\mathrm{sI}-\mathrm{A}]^{-1} \mathrm{~B}$ u

where $B=\left[\begin{array}{lll}1 & 0 & 0\end{array}\right]^{\mathrm{T}}$

$[\mathrm{sI}-\mathrm{A}]^{-1}$

$=\frac{1}{\Delta}\left[\begin{array}{lll}\left(\mathrm{s}+\mathrm{k}_{12}+\mathrm{k}_{32}+\mathrm{k}_{02}\right)\left(\mathrm{s}+\mathrm{k}_{23}\right)-\mathrm{k}_{32} \mathrm{k}_{23} & \mathrm{k}_{12}\left(\mathrm{~s}+\mathrm{k}_{23}\right) \mathrm{k}_{12} \mathrm{k}_{23} \\ \mathrm{k}_{21}\left(\mathrm{~s}+\mathrm{k}_{23}\right) & \left(\mathrm{s}+\mathrm{k}_{21}\right)\left(\mathrm{s}+\mathrm{k}_{23}\right) & \left(\mathrm{s}+\mathrm{k}_{21}\right) \mathrm{k}_{23} \\ \mathrm{k}_{21} \mathrm{k}_{32} & \left(\mathrm{~s}+\mathrm{k}_{21}\right) \mathrm{k}_{32}\left(\mathrm{~s}+\mathrm{k}_{21}\right) & \left(\mathrm{s}+\mathrm{k}_{12}+\mathrm{k}_{32}+\mathrm{k}_{02}\right)-\mathrm{k}_{12} \mathrm{k}_{21}\end{array}\right]$

where $\Delta=\operatorname{det}[\mathrm{s} I-\mathrm{A}]$

$\mathrm{k}_{12} \mathrm{k}_{23}$ $=s^{3}+s^{2}\left(k_{21}+k_{12}+k_{32}+k_{02}+k_{23}\right)+s\left(k_{21} k_{32}+k_{21} k_{02}+k_{21} k_{23}+\right.$

$$
\left.+\mathrm{k}_{23} \mathrm{k}_{02}\right)+\mathrm{k}_{2 \mathrm{i}} \mathrm{k}_{23} \mathrm{k}_{02}
$$

$\mathrm{x}=\frac{1}{\Delta}\left[\begin{array}{l}\mathrm{s}^{2}+\mathrm{s}\left(\mathrm{k}_{12}+\mathrm{k}_{32}+\mathrm{k}_{02}+\mathrm{k}_{23}\right)+\mathrm{k}_{12} \mathrm{k}_{23}+\mathrm{k}_{23} \mathrm{k}_{02} \\ \mathrm{k}_{21}\left(\mathrm{~s}+\mathrm{k}_{23}\right) \\ \mathrm{k}_{21} \mathrm{k}_{32}\end{array}\right]$

$C=\left[\begin{array}{lll}\frac{1}{V_{1}} & 0 & 0\end{array}\right]^{\mathrm{T}}$ where $\mathrm{V}_{1}$ is the intitial volume of distribution (plasma volume)

$$
\begin{aligned}
& y=C^{\top} x \\
& y=\frac{s^{2}+s\left(k_{12}+k_{32}+k_{02}+k_{23}\right)+k_{12} k_{23}+k_{23} k_{02}}{\Delta V_{1}}
\end{aligned}
$$

The steady state plasma ${ }^{14} \mathrm{C}$ leucine concentration following a unit step input is

$\mathrm{y}=\frac{\mathrm{k}_{12}+\mathrm{k}_{02}}{\mathrm{~V}_{1} \mathrm{k}_{21} \mathrm{k}_{02}}$

$\mathrm{MCR}=\frac{1}{\mathrm{y}}=\frac{\mathrm{V}_{1} \mathrm{k}_{21} \mathrm{k}_{02}}{\mathrm{k}_{12}+\mathrm{k}_{02}}$

$\mathrm{Ra}$ (leucine production rate) $=\frac{\mathrm{V}_{1} \mathrm{k}_{21} \mathrm{k}_{02} \text { [leucine] }}{\mathrm{k}_{12}+\mathrm{k}_{02}}$

where [leucine] $=$ steady state plasma leucine concentration be

The solution of the output equation (A12) in the time domain will

$y(t)=A_{1} \mathrm{e} \quad \lambda 1 \mathrm{t}+\mathrm{A}_{2} \mathrm{e} \quad \lambda 2 \mathrm{t}+\mathrm{A}_{3} \mathrm{e} \quad \lambda 3 \mathrm{t}$

or in the $s$ domain

$y=\frac{A_{1}}{s+\lambda_{1}}+\frac{A_{2}}{s+\lambda_{2}}+\frac{A_{3}}{s+\lambda_{3}}$

by equating the coefficient of $\mathrm{s}$ in (A17) with (A12) and the common denominator of (A17) with (A9), the following equations would exist

$\mathrm{k}_{12}+\mathrm{k}_{32}+\mathrm{k}_{02}+\mathrm{k}_{23}=\mathrm{A}$

$\mathrm{k}_{12} \mathrm{k}_{23}+\mathrm{k}_{23} \mathrm{k}_{02}=\mathrm{B}$

$\mathrm{k}_{12}+\mathrm{k}_{32}+\mathrm{k}_{02}+\mathrm{k}_{23}+\mathrm{k}_{21}=\mathrm{C}$ $k_{21} k_{32}+k_{21} k_{02}+k_{21} k_{23}+k_{23} k_{12}+k_{23} k_{02}=D$

$\mathrm{k}_{21} \mathrm{k}_{23} \mathrm{k}_{02}=\mathrm{E}$

where $A, B, C, D$ and $E$ are functions of the fitted curve parameters.

From [A 18] and [A 20], $k_{21}$ is determined. Examination of equations [A 19] to [A22] indicates that this three compartmental model (catenary) is uniquely identifiable.

The plasma leucine concentration can be simulated by using the estimated production rate $(\mathrm{Ra})$ as the input to compartment 1 , since this is the production rate as seen entering the plasma compartments. The initial conditions for compartments 1 to 3 are

$\mathrm{x}_{1}(0)=\mathrm{V}_{1}$ [leucine]

$\mathrm{x}_{2}(0)=\frac{\mathrm{x}_{1}(0) \mathrm{k}_{21}-\mathrm{Ra}}{\mathrm{k}_{12}}$

$\mathrm{x}_{3}(0)=\frac{\mathrm{x}_{2}(0) \mathrm{k}_{32}}{\mathrm{k}_{23}}$

The production rates, as seen entering compartment 2 or 3 with measurements made in compartment 1 , are identical in the steady state. These estimates are derived using Eq. (A6)-(A15) with B=[010] and $B=[001]$ for $\mathrm{Ra}$ entering compartment 2 and 3 respectively. The estimate of production rate is:

$\mathrm{Ra}$ (leucine production rate) $=\frac{\mathrm{V}_{1} \mathrm{k}_{21} \mathrm{k}_{02} \text { [leucine] }}{\mathrm{k}_{12}}$

The bicarbonate subsystem was shown to be an adequate description of bicarbonate metabolism following an injection of $\mathrm{NaH}^{14} \mathrm{CO}_{3}$ in 17 control subjects [42]. The mean model parameters and the range of values calculated in these 17 subjects are shown in Table 6.

The link between the leucine and bicarbonate subsystems, $\mathrm{k}_{42}$, was determined by simulating the full 6 compartment model for 4 of the control subjects $[1,6,7$ and 9$]$ in whom the bicarbonate subsystem model had been uniquely identified from bicarbonate kinetics obtained following the injection of $\mathrm{NaH}^{14} \mathrm{CO}_{3}$ into compartment 5 . Since for these subjects $\mathrm{k}_{02}$ was known from identification of leucine subsystems, $k_{p 2}$ and $k_{42}$ could then be determined by calculating the percentage leucine oxidation from the equation:

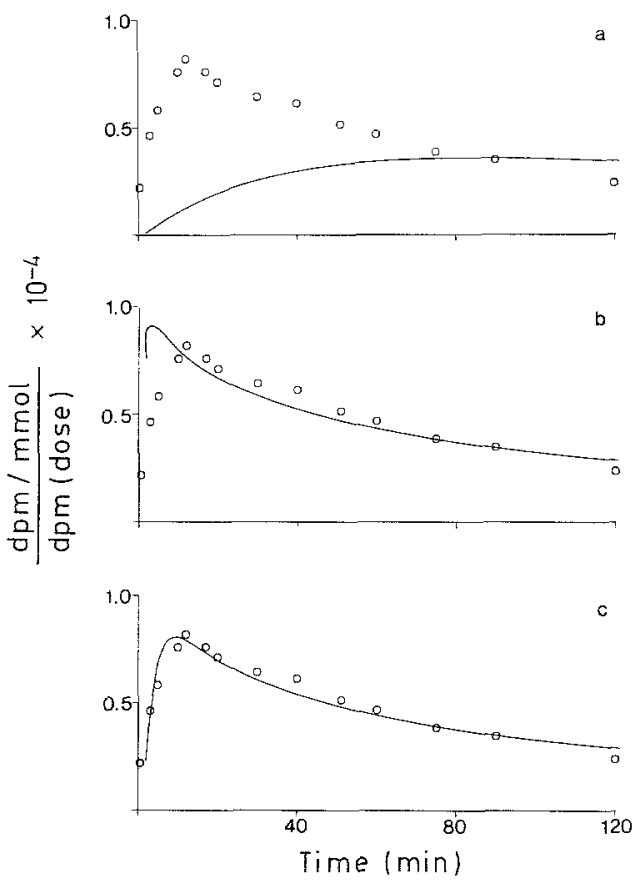

Fig. 6. Experimental breath ${ }^{14} \mathrm{CO}_{2}$ data $(O)$ and the predicted curves produced by simulation of the total model $(-)$ in control subject 7 with links between (a) compartments 2 and 6 (b) compartments 2 and 5 and (c) compartments 2 and 4 
$\frac{\text { \% oxidation }}{100}=\frac{\text { area under } \mathrm{CO}_{2} \text { specific activity curve }\left(\mathrm{dpm} \mathrm{mmol} \mathrm{m}^{-1} \mathrm{~min}\right) \times \mathrm{CO}_{2} \text { production rate }(\mathrm{mmol} / \mathrm{min})}{\mathrm{k}_{\mathrm{p}}+\mathrm{k}_{42}}$ 100 total dose adminstered (dpm)

The area under the $\mathrm{CO}_{2}$ specific activity curve is derived using the sum of two exponentials with a least square fit to the experimental data. The model ${ }^{14} \mathrm{CO}_{2}$ output was found to match the experimental output when compartment 2 of the leucine subsystem was linked to compartment 4 of the bicarbonate subsystem as shown for subject 7 in Figure 6.

In the remaining diabetic patients and control subjects best estimates of parameters $\mathrm{k}_{42}$ and the 5 parameters for the bicarbonate subsystem were determined by a process of adaptive fitting [43] minimising the sum of squares error between model derived and experimental $\mathrm{CO}_{2}$ specific activity curves. Initial estimates for the bicarbonate parameters and upper and lower limits between which parameters could vary were taken from parameters found in control subjects (Table 6). The match between the model derived and experimental ${ }^{14} \mathrm{CO}_{2}$ curve using the fitting procedure is demonstrated in diabetic patient 7 following insulin withdrawal, conventional therapy and an insulin infusion in Figure 7 .

To check the validity of model parameter estimates $\mathrm{CO}_{2}$ production rates, calculated using parameter $\mathrm{k}_{05}$ in output equation [11], were compared with experimentally determined $\mathrm{CO}_{2}$ production rates (Table 7) and were found to be in close agreement.

Acknowledgements. We would like to thank Ms. S. Prestwich for her excellent technical assistance, Ms. L. Lawrence for her help with the manuscript, and the British Diabetic Association, The Medical Research Council and St Thomas' Hospital Endowment Committee for funding this research.
Table 6. Mean bicarbonate model parameters calculated from the expired ${ }^{14} \mathrm{CO}_{2}$ curve following injection of $\mathrm{NaH}^{14} \mathrm{CO}_{3}$ in 17 control subjects

\begin{tabular}{lll}
\hline Model parameter & $\begin{array}{l}\text { Mean } \pm \mathrm{SD} \\
\left(\mathrm{min}^{-1}\right)\end{array}$ & $\begin{array}{l}\text { Range } \\
\left(\mathrm{min}^{-1}\right)\end{array}$ \\
\hline $\mathrm{K}_{45}$ & $1.0273 \pm 0.768$ & $0.2029-2.378$ \\
$\mathrm{k}_{54}$ & $0.6398 \pm 0.551$ & $0.1327-1.562$ \\
$\mathrm{k}_{65}$ & $0.2302 \pm 0.089$ & $0.1137-0.4568$ \\
$\mathrm{k}_{56}$ & $0.0570 \pm 0.052$ & $0.0088-0.0662$ \\
$\mathrm{k}_{05}$ & $0.0980 \pm 0.025$ & $0.0649-0.1438$ \\
\hline
\end{tabular}

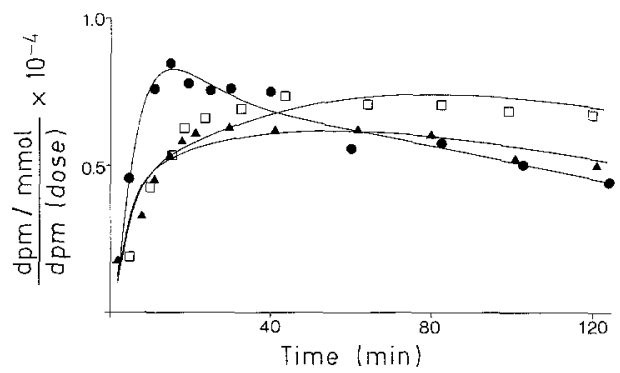

Fig.7. Model derived breath ${ }^{14} \mathrm{CO}_{2}$ specific activity time-curves and experimental data points in a diabetic patient 7 following insulin withdrawal $(\square)$, conventional therapy $(\Delta)$ and an insulin infusion (อ)

Table 7. Mean model derived and measured $\mathrm{CO}_{2}$ production rate in diabetic patients and control subjects

\begin{tabular}{|c|c|c|c|c|c|c|c|c|}
\hline & \multicolumn{6}{|c|}{ Diabetic patients (mmol/min) } & & \\
\hline & \multicolumn{2}{|c|}{ Insulin withdrawal } & \multicolumn{2}{|c|}{ Conventional therapy } & \multicolumn{2}{|c|}{ Insulin infusion } & \multicolumn{2}{|c|}{ Control subjects } \\
\hline & Model & Measured & Model & Measured & Model & Measured & Model & Measured \\
\hline $\begin{array}{l}\text { Mean } \\
\pm \text { SEM }\end{array}$ & $\begin{array}{r}9.93 \\
\pm 0.56\end{array}$ & $\begin{array}{r}9.01 \\
\pm 0.61\end{array}$ & $\begin{array}{r}8,79 \\
\pm 0.76\end{array}$ & $\begin{array}{r}8.40 \\
\pm 0.65\end{array}$ & $\begin{array}{r}9.29 \\
\pm 0.89\end{array}$ & $\begin{array}{r}9.26 \\
\pm 0.80\end{array}$ & $\begin{array}{r}10.89 \\
\pm 0.87\end{array}$ & $\begin{array}{r}11.65 \\
\pm 0.56\end{array}$ \\
\hline
\end{tabular}

\section{References}

1. Felig P, Marliss E, Ohman JL, Cahill GF Jr (1970) Plasma amino acids in diabetic ketoacidosis. Diabetes 19: 727-729

2. Berger M, Zinnerman-Telschow H, Berchtold P, Drost H, Muller WA, Gries FA, Zimmerman H (1978) Blood amino acid levels in patients with insulin excess (functioning insulinoma) and insulin deficiency (diabetic ketosis). Metabolism 27:793-799

3. Brown PM, Umpleby AM, Meek J, Prestwich SA, Sönksen PH (1980) Abnormalities of plasma amino acid concentrations in insulin-deprived diabetic patients and the effect of low and high dose infusions of insulin. Diabetologia 19: 261

4. Tamborlane WV, Sherwin RS, Genel M, Felig P (1979) Restoration of normal lipid and amino acid metabolism in diabetic patients treated with a portable insulin infusion pump. Lancet 1 : $1258-1261$

5. Miller LL (1961) In: Holden JT (ed) Amino acid pools. Elsevier, Amsterdam, pp 708-721

6. Ruderman NB, Berger M (1974) The formation of glutamine and alanine in skeletal muscle. J Biol Chem 249: 5500-5506

7. Buse MG, Werlong HF, Weigand DA (1976) The effect of diabetes, insulin and the redox potential on leucine metabolism by isolated rat hemidiaphragm. Endocrinology 98: 1166-1175
8. Paul HS, Adibi SA (1978) Leucine oxidation in diabetes and starvation: effects of ketone bodies on branched-chain amino acid oxidation in vitro. Metabolism 27:185-200

9. Wahren J, Felig P, Cerasi E, Luft R (1972) Splanchnic and peripheral glucose and amino acid metabolism in diabetes mellitus. $J$ Clin Invest 51: 1870-1878

10. Sherwin RS, Rosenzweig J, Soman V, Hendler RG, Felig P (1976) Effect of insulin and diabetes on protein and branched-chain amino acid utilization. Diabetes 25: 332

11. Saunders J, Boroujerdi MA, Brown PM, Carson ER, Hall SEH, Umpleby AM, Sönksen PH (1982) Isotope turnover studies in uncontrolled diabetes and the effects of insulin. In: Porter R, Lawrenson $\mathbf{G}$ (eds) Metabolic acidosis. (Ciba Foundation Symposium 87) Pitman, London, pp 273-292

12. Williamson DH, Mellanby J, Krebs HA (1962) Enzymic determination of $\mathrm{D}(-)$ hydroxybutyric acid and acetoacetic acid in blood. Bichemistry 82:90-96

13. Hohorst HJ, Kreutz FH, Bücher T (1959) Über Metabolitgehalte und Metabolit-Konzentrationen in der Leber der Ratte. Biochem Z 332: 18-46

14. Dole VP, Meinertz H (1960) Microdetermination of long-chain fatty acids in plasma and tissues. J Biol Chem 235: 2595-2599 
15. Carruthers M, Young DAB (1973) Free fatty acid estimation by a semi-automated fluorimetric method. Clin Chim Acta 49: 341-348

16. Baron MD, Shenouda F, Sönksen PH (1980) A micro-column method for $\mathrm{HbA}_{\mathrm{fc}}$ determination. Lancet 2: 1114-1116

17. Marquardt DW (1963) An algorithm for least-squares estimation of non-linear parameters. J Soc Indust Appl Math 11: 431-441

18. Godfrey KR (1983) Some practical problems of compartmental modelling. In: Compartmental models and their application. Academic, London, pp 146-180

19. Sterling K, Gray SJ (1950) Determination of the circulating red cell volume in man by radioactive chromium. J Clin Invest 29: 1614-1619

20. McKee EE, Cheung JY, Rannels DE, Morgan HE (1978) Measurement of the rate of protein synthesis and compartmentation of heart phenylalanine. J Biol Chem 253: 1030-1040

21. Mortimore GE, Woodside KH, Henry JE (1972) Compartmentation of free valine and its relation to protein turnover in perfused rat liver. J Biol Chem 247: 2776-2784

22. Steele $\mathbf{R}$ (1955) The retention of metabolic radioactive carbonate. Biochem J 60: 447-453

23. Waterhouse C, Baker N, Rostami H (1969) Effect of glucose ingestion on the metabolism of free fatty acids in human subjects. J Lipid Res 10: 487-494

24. Corbelli C, DiStefano JJ III (1980) Parameter and structural identifiability concepts and ambiguities, a critical review and analysis. Am J Physiol 239: 7-24

25. DiStefano JJ III (1982) Non-compartmental versus compartmental analysis: some basis for choice. Am J Physiol 243: 1-6

26. Snedecor GW, Cochran WG (1976) Statistical Methods 6th Edition. State University Press Ames Iowa

27. Nair KS, Garrow JS, Ford C, Mahler RF, Halliday D (1983) Effect of poor diabetic control and obesity on whole body protein metabolism in man. Diabetologia 25: 400-403

28. Rennie MJ, Matthews DE, Bier DM, Halliday D, Clugston GC, Edwards RHT, Millward DJ (1980) Enrichment of muscle free amino acids during infusion of $1-{ }^{13} \mathrm{C},{ }^{15} \mathrm{~N}$ leucine. Eur $\mathrm{J}$ Clin Invest 10:31 (Abstract)

29. Matthews DE, Schwarz HP, Young RD, Motil KJ, Young VR, Bier DM (1982) Relationship of plasma leucine and alpha-ketoisocaproate during a $L-\left[1-{ }^{13} \mathrm{C}\right]$ leucine infusion in man: a method for measuring human intracellular leucine tracer enrichment. Metabolism 31: 1105-1112

30. Pain VM, Garlick PJ (1974) Effect of streptozotocin diabetes and insulin treatment on the rate of protein synthesis in the tissues of the rat in vivo. $\mathrm{J}$ Biol Chem $249: 4510-4514$

31. Williams IH, Chua BHL, Sahms RH, Siehl D, Morgan HE (1980) Effects of diabetes on protein turnover in diabetic muscle. Am J Physiol 239: E178-E185
32. Garlick PJ, Albertse EC, McNurlan MA, Pain BM (1981) Protein turnover in tissues of diabetic rats. Acta Biol Med Germ 40: 1301-1307

33. Jefferson LS, Liao WSL, Peavy DE, Miller CB, Appel MC, Taylor JM (1983) Diabetes induced alterations in liver protein synthesis. J Biol Chem 258: 1369-1375

34. Goodman HM, Frick GP (1981) Metabolism of branched chain amino acids in adipose tissue. In: Walser $M$, Williamson JR (eds) Metabolism and clinical implications of branched chain amino and keto acids. Elsevier North Holland, New York Oxford Shannon, pp $169-180$

35. Odessey R, Khairallah EA, Goldberg AL (1974) Origin and possible significance of alanine production by skeletal muscle. $\mathrm{J}$ Biol Chem 249: 7623-7629

36. Chang TW, Goldberg AL (1978) The origin of alanine produced in skeletal muscle. J Biol Chem 253:3677-3684

37. Palaiologos G, Felig $P$ (1976) Effects of ketone bodies on amino acid metabolism in isolated rat diaphragm. Biochem $J$ 154: 709-716

38. Sherwin RS, Hendler RG, Felig P (1975) Effect of ketone infusions on amino acid and nitrogen metabolism in man. J Clin Invest 55: $1382-1390$

39. Hall SEM, Braaten JT, McKendry JBR, Bolton T, Foster D, Berman M (1979) Normal alanine-glucose relationships and changes in diabetic patients before and after insulin treatment. Diabetes 28: 737-744

40. Haymond M, Tessari P, Beausrere B, Tsalikian E, Miles J (1985) Effect of fatty acid availability on leucine metabolism in vivo. In: Garrow JS, Halliday D (eds) Substrate and energy metabolism in man (3rd CRC International Symposium). John Libbey, London Paris, pp 179-186

41. Hedden MP, Buse MG (1982) Effects of glucose, pyruvate, lactate and amino acids on muscle protein synthesis. Am J Physiol 242: E184-E192

42. Umpleby AM, Boroujerdi MA, Brown PM, Carson ER, Sönksen PH (1981) A compartment model of leucine and bicarbonate metabolism in man. Clin Sci 61: 45P

43. Gill PE, Murray W (1976) Minimization subject to bounds on the variables. National Physical Laboratory Report no NAC 72. Her Majesty's Stationery Office, London

Received: 1 February 1985

and in revised form: 16 December 1985

Dr. A. M. Umpleby

Department of Medicine

St Thomas' Hospital

Lambeth Palace Road

London SE1 7EH, UK 